University of Nebraska - Lincoln

DigitalCommons@University of Nebraska - Lincoln

8-2008

\title{
Generation of Stable, Low-Divergence Electron Beams by Laser- Wakefield Acceleration in a Steady-State-Flow Gas Cell
}

\author{
J. Osterhoff \\ Max-Planck-Institut für Quantenoptik
}

A. Popp

Max-Planck-Institut für Quantenoptik

Zs. Major

Max-Planck-Institut für Quantenoptik

B. Marx

Max-Planck-Institut für Quantenoptik

T.P. Rowlands-Ree

University of Oxford

See next page for additional authors

Follow this and additional works at: https://digitalcommons.unl.edu/physicsfuchs

Osterhoff, J.; Popp, A.; Major, Zs.; Marx, B.; Rowlands-Ree, T.P.; Fuchs, Matthias; Geissler, M.; Horlein, R.; Hidding, B.; Becker, S.; Peralta, E.A.; Schramm, U.; Gruner, F.; Habs, D.; Krausz, F.; Hooker, Simon M.; and Karsh, Stefan, "Generation of Stable, Low-Divergence Electron Beams by Laser-Wakefield Acceleration in a Steady-State-Flow Gas Cell" (2008). Matthias Fuchs Publications. 4.

https://digitalcommons.unl.edu/physicsfuchs/4

This Article is brought to you for free and open access by the Research Papers in Physics and Astronomy at DigitalCommons@University of Nebraska - Lincoln. It has been accepted for inclusion in Matthias Fuchs Publications by an authorized administrator of DigitalCommons@University of Nebraska - Lincoln. 


\section{Authors}

J. Osterhoff, A. Popp, Zs. Major, B. Marx, T.P. Rowlands-Ree, Matthias Fuchs, M. Geissler, R. Horlein, B. Hidding, S. Becker, E.A. Peralta, U. Schramm, F. Gruner, D. Habs, F. Krausz, Simon M. Hooker, and Stefan Karsh 


\title{
Generation of Stable, Low-Divergence Electron Beams by Laser-Wakefield Acceleration in a Steady-State-Flow Gas Cell
}

\author{
J. Osterhoff, ${ }^{1}$ A. Popp, ${ }^{1}$ Zs. Major, ${ }^{1,2}$ B. Marx, ${ }^{1,2}$ T. P. Rowlands-Rees,${ }^{3}$ M. Fuchs,${ }^{1,2}$ M. Geissler, ${ }^{4}$ R. Hörlein, ${ }^{1,2}$ \\ B. Hidding, ${ }^{5}$ S. Becker, ${ }^{2}$ E. A. Peralta, ${ }^{1}$ U. Schramm, ${ }^{6}$ F. Grüner, ${ }^{1}$ D. Habs,${ }^{2}$ F. Krausz, ${ }^{1,2}$ S. M. Hooker, ${ }^{3}$ and S. Karsch ${ }^{1, *}$ \\ ${ }^{1}$ Max-Planck-Institut für Quantenoptik, 85748 Garching, Germany \\ ${ }^{2}$ Sektion Physik, Ludwig-Maximilians-Universität München, 85748 Garching, Germany \\ ${ }^{3}$ Clarendon Laboratory, University of Oxford, OX1 3PU, United Kingdom \\ ${ }^{4}$ Centre for Plasma Physics, Queen's University Belfast, BT7 1NN, United Kingdom \\ ${ }^{5}$ Institut für Laser- und Plasmaphysik, Heinrich-Heine-Universität Düsseldorf, 40225 Düsseldorf, Germany \\ ${ }^{6}$ Forschungszentrum Dresden-Rossendorf, 01328 Dresden, Germany
}

(Received 14 April 2008; published 21 August 2008)

\begin{abstract}
Laser-driven, quasimonoenergetic electron beams of up to $\sim 200 \mathrm{MeV}$ in energy have been observed from steady-state-flow gas cells. These beams emitted within a low-divergence cone of $2.1 \pm 0.5 \mathrm{mrad}$ FWHM display unprecedented shot-to-shot stability in energy $(2.5 \% \mathrm{rms})$, pointing $(1.4 \mathrm{mrad} \mathrm{rms})$, and charge $(16 \% \mathrm{rms})$ owing to a highly reproducible gas-density profile within the interaction volume. Laserwakefield acceleration in gas cells of this type provides a simple and reliable source of relativistic electrons suitable for applications such as the production of extreme-ultraviolet undulator radiation.
\end{abstract}

DOI: 10.1103/PhysRevLett.101.085002

PACS numbers: $52.38 . \mathrm{Kd}, 41.75 . \mathrm{Jv}, 52.38 . \mathrm{Hb}$

In recent years, laser-wakefield acceleration (LWFA) has advanced at a remarkable pace. The vision of a laserdriven plasma-based electron accelerator [1] started to become reality when multi-MeV laser-accelerated electrons were first detected in the mid-1990s [2]. Since then, an improvement in technology and understanding has led to such breakthroughs as the generation of quasimonoenergetic electron beams [3,4] in the bubble regime [5], the extension of electron energies into the $\mathrm{GeV}$ range [6,7], and their systematic manipulation by means of controlled injection [8]. These achievements pave the way towards practical applications. In the future, this accelerator concept could permit tabletop drivers for extreme-ultraviolet and x-ray free-electron lasers [9]. For these purposes, extensive control over electron bunch reproducibility and quality is of crucial importance, progress on which is reported in this Letter.

In LWFA, the ponderomotive force of a laser pulse traveling through underdense plasma can excite a plasma wave with longitudinal electric fields larger than $10 \mathrm{GV} / \mathrm{m}$. These fields may be utilized to accelerate electrons to ultrarelativistic energies on a centimeter scale. For this process to occur, the electrons need to acquire an initial momentum to be injected into the wakefield structure. This can be facilitated by the laser pulse itself when its intensity reaches the injection threshold, which depends on the electron plasma density $n_{e}$. That mechanism is most efficient when the longitudinal laser size $L=c \tau$, with pulse duration $\tau$ and the speed of light $c$, is comparable to its transverse dimensions and approximately equal to half the plasma wavelength $\lambda_{P}=2 \pi c / \omega_{p}$ [10], with $\omega_{P}$ being the plasma frequency. However, even a laser pulse that initially does not satisfy these conditions can potentially be matched to these constraints by self-compression [11] and relativistic self-focusing $[12,13]$, with the latter taking place above a critical laser power $P_{\text {crit }} \geqslant 17\left(\omega_{L} / \omega_{P}\right)^{2} \mathrm{GW}$. Here $\omega_{L}$ represents the laser frequency. Owing to a nonlinear dependence on the laser and plasma parameters, both pulse-shaping effects may cause significant fluctuations in electron-beam properties due to small shot-to-shot variations in laser and plasma conditions. Most LWFA experiments published to date have relied to some extent on nonlinear laser-pulse evolution prior to the acceleration process and at the same time demonstrated only limited stability (with the exception of Ref. [8]). Hence, for delivering high-quality and stable electron bunches that allow for applications, it is mandatory to minimize fluctuations of the laser as well as the plasma parameters and thus reduce the consequences of nonlinearities. Lately, a number of studies have been dedicated to improving control over electron beam reproducibility. Stabilizing the bunch energy has been demonstrated in a complex two-beam setup [8]. Other groups have analyzed the laser pulse and plasma-parameter space to optimize acceleration stability $[6,14,15]$. In this Letter, we report on the generation of high-quality laser-accelerated electron beams of unprecedented simultaneous stability in energy, charge, divergence, and pointing direction. The presented experiment is based on a simple setup using a stable gas-filled capillary target and thereby minimizes shot-to-shot fluctuations. In contrast to previous work employing such a design [6,7], the target gas is ionized by the wake-driving laser pulse and not by an electrical discharge.

The gas cell was constructed from two sapphire plates, each with a half-cylindrical groove in its surface. The grooves were aligned to form a $15 \mathrm{~mm}$ long, $250 \mu \mathrm{m}$ diameter channel. These channels are long-lived, since wall erosion by the laser has no detectable influence on 
the electron beams. Experiments with different diameter gas cells of 200-300 $\mu \mathrm{m}$ yielded similar results, suggesting that unlike in Ref. [6] the waveguide properties of these structures do not play a prominent role here. Figure 1(a) shows the cell cross section and a sketch of the experimental setup. The channel was filled with hydrogen passing through a valve and two gas slots at backing pressures of up to 490 mbar. We carried out simulations of the gas flow using the 2D coupled-implicit-solver version of the FLUENT code, which solves the Navier-Stokes equations and in our case uses the $\kappa-\varepsilon$ model to account for turbulence [16]. The gas cell investigated in this work reduces target density fluctuations threefold compared to supersonic gas jets. First, negligible gas flow occurs within the central interaction volume. The magnified regions in Fig. 1 (a) display the steady-state flow velocities at the cell entrance or exit and center for a typical backing pressure of 130 mbar. An almost perfectly homogeneous and stationary gas distribution develops between the inlets covering $80 \%$ of the channel length [Fig. 1(b)]. In addition, the residual flow at the cell exits and supply slots is only marginally turbulent, and the turbulent kinetic energy $\kappa$ is low. Second, no supersonic shock fronts propagate through the medium, a phenomenon which may occur in high-Mach gas jets. Finally, the valve opening process has no influence on the actual density distribution inside the channel since the time it takes the valve to open $(\sim 2 \mathrm{~ms})$ is 2 orders of magnitude smaller than the cell filling time in pulsed operation $(200 \mathrm{~ms})$ and is irrelevant in continuous-flow mode. A destabilization of the electron-acceleration conditions was observed only for valve opening times below $\sim 50 \mathrm{~ms}$. Thus, initial fluctuations equilibrate before the arrival of the laser pulse and a steady-state, laminar gas
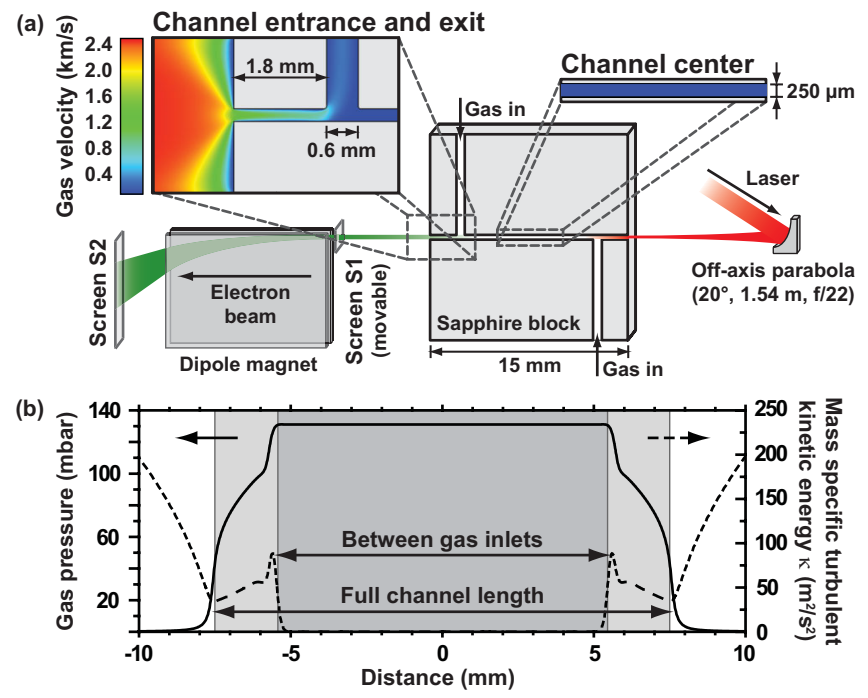

FIG. 1 (color). (a) Cross section of a capillary. Magnified parts: Color-coded gas-flow velocities in a steady-state regime (FLUENT simulation with 130 mbar filling pressure). (b) Calculated gas-pressure profile (solid line) and the turbulent kinetic energy $\kappa$ (dashed line) along the central channel axis. flow arises resulting in a reproducible, homogeneous gas distribution.

The driver laser in our experiment, the ATLAS Ti:sapphire system at the Max-Planck-Institut für Quantenoptik, delivered $20 \mathrm{TW}, 850 \mathrm{~mJ}, 42 \mathrm{fs}$ FWHM pulses on target at a central wavelength of $\lambda \approx 800 \mathrm{~nm}$. As schematically indicated in Fig. 1(a), the beam was then focused into the gas cell by an $f / 22$ off-axis parabola to a spot with a diameter of $23 \mu \mathrm{m}$ FWHM and a Strehl ratio of $>0.7$. This resulted in an average vacuum FWHM focal intensity of $1.7 \times 10^{18} \mathrm{~W} \mathrm{~cm}^{-2}$ and a normalized laser vector potential of $a_{0}=0.89$.

The accelerated electrons emerging from the gas channel were observed at two positions along their trajectories by 12-bit cameras on scintillating phosphor screens (type CAWO OG 16). These screens were placed at the entrance of $(S 1)$ and behind $(S 2)$ a permanent dipole magnet (field strength of $\sim 0.45 \mathrm{~T}$ ). $S 1$ detected the electron beam pointing and divergence after $1.12 \mathrm{~m}$ of propagation. When this screen was removed, the electrons could enter the spectrometer magnet unscattered. $S 2$ then registered the electron deflection in the magnetic field. The fluorescence signal of $S 2$ was calibrated using image plates to provide an absolute charge readout [17]. Three-dimensional particle tracking simulations for the measured field distribution of the spectrometer were used to calibrate its energy dispersion and transverse focusing behavior. We have investigated the electron beams generated from this setup in terms of charge, energy, pointing direction, and divergence. The remarkable shot-to-shot stability of these parameters allowed for systematic studies with meaningful statistics which will be presented in the following.

Figure 2 displays the negative charge $Q$ of accelerated particles measured at $S 2$ as a function of $n_{e}$ with energies $\geq 100 \mathrm{MeV}$. The electron density is deduced from the hydrogen fill pressure assuming complete ionization within the laser focus. Electrons are injected into the wake at densities above $\sim 4 \times 10^{18} \mathrm{~cm}^{-3}$ facilitated by laser self-modulation effects (see remarks on simulations below). At $n_{e}^{\prime} \approx 7.3 \times 10^{18} \mathrm{~cm}^{-3}$, a maximum amount of charge $Q \approx 32 \mathrm{pC}$ is detected with shot-to-shot fluctuations as low as $16 \%$ rms. The data on either side of this maximum can be interpreted as follows: For densities $n_{e}<$ $n_{e}^{\prime}$, an increase towards $n_{e}^{\prime}$ in charge and stability is observed. In this regime, the distance $d$ over which injected electrons interact with the wakefield is governed by the focusing geometry of the laser modified through relativistic self-focusing and energy depletion effects and not by the electron dephasing length $l_{d}>d \quad\left(l_{d}=\lambda_{p}^{3} / \lambda^{2} \propto\right.$ $n_{e}^{-3 / 2}$ in linear theory). $l_{d}$ is the distance over which the electrons outrun the accelerating wakefield and start to decelerate. The laser energy depletion length $l_{e} \propto n_{e}^{-1}$ [18] decreases with density as fast as $P_{\text {crit }} \propto n_{e}^{-1}$. Therefore, the distance over which the self-focusing effect can be maintained does not depend on density resulting in an almost constant acceleration distance. Since the electric 


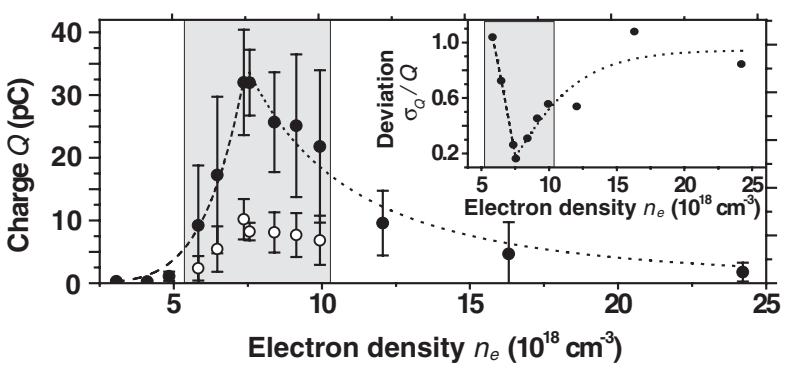

FIG. 2. The negative charge $Q$ of high-energy electrons ( $>$ $100 \mathrm{MeV}$ ) as a function of the plasma density $n_{e}$. Each data point is averaged over up to 50 shots. The inset displays the relative shot-to-shot standard deviation $\sigma_{Q}$ of charge $Q$ vs $n_{e}$. Empty circles represent the charge contained within the peaks. Shaded areas: Electron beams are predominantly quasimonoenergetic.

field of the wake $E_{w}$ is proportional to $n_{e}^{1 / 2}$, the electron energy $W_{e} \propto E_{w} d$ increases accordingly as does the number of electrons above $100 \mathrm{MeV}$. In addition, a more efficient electron trapping with higher density increases the total amount of accelerated charge. With $n_{e}$ approaching $n_{e}^{\prime}$, the dephasing length converges to $d$ with $l_{d} \approx$ $4 \mathrm{~mm}$, representing the longest distance over which electrons are accelerated and setting a lower limit on the length over which the laser pulse is self-guided. This constitutes the transition to a dephasing-limited regime for densities $n_{e}>n_{e}^{\prime}$, in which $l_{d}<d$ and $W_{e} \propto E_{w} l_{d} \propto 1 / n_{e}$. Thus, $Q$ decreases with higher density. Alongside dephasing, a lower beam-loading threshold with increasing plasma density diminishes the total amount of accelerated charge. The decreased stability for $n_{e}>n_{e}^{\prime}$ can be ascribed to a growing deviation from the LWFA resonance condition $\lambda_{p} / L \approx$ 2 , leading to filamentation and temporal laser breakup.
The onset of the filamentation processes can be observed in some of the energy-resolved electron beams obtained at $n_{e}^{\prime}$ [cf. Fig. 3(a)]. False-color images of $S 2$ show the dispersed bunches accelerated by 40 consecutive laser shots. It may be seen that every shot led to electron acceleration. The electron spectra in this regime exhibit a plateau background of $22 \pm 5 \mathrm{pC}$ with a quasimonoenergetic peak containing $10 \pm 4 \mathrm{pC}$ of charge at $198 \pm$ $12 \mathrm{MeV}$ appearing in $80 \%$ of the cases [Fig. 3(b)]. These features are stable in energy with a standard deviation of $6 \%$ for the electron peak and 3\% for the high-energy cutoff at $217 \pm 6 \mathrm{MeV}$. The energy spread of the peak $\Delta E / E$ amounts to $8.2 \%$ FWHM, with $\Delta E \approx 16.3 \mathrm{MeV}$.

At a decreased density of $n_{e} \approx 6.8 \times 10^{18} \mathrm{~cm}^{-3}$, the plateau vanishes, and only the quasimonoenergetic part remains [Fig. 3(c)]. This comes at the expense of a reduced peak charge $(7 \pm 3 \mathrm{pC})$, reduced electron energy (122 \pm $3 \mathrm{MeV}$ ), and a reduced injection probability (71\%), which indicates that the laser pulses are only just above the injection threshold. Remarkably, the peak energy stability improves to $2.5 \% \mathrm{rms}$. To our knowledge, this is the best shot-to-shot reproducibility in an LWFA experiment achieved to date. The remaining variations in the acceleration process can be attributed not only to residual gas fluctuations, laser shot-to-shot jitter in energy $(\leq 2.0 \%$ rms), and pulse duration but also to electron pointing deviations into the dipole magnet introducing an uncertainty, which will be investigated in the following.

The accumulated signal of 74 electron bunches and the locations of their maxima on $S 1$ are presented in Fig. 4(a). Here the beam pointing within the spectrometer deflection plane varies by $1.4 \mathrm{mrad} \mathrm{rms}$. For a fixed electron energy, this fluctuation translates into apparent energy fluctuations on screen $S 2$ with standard deviations of $1.7 \%$ at $122 \mathrm{MeV}\left(n_{e} \approx 6.8 \times 10^{18} \mathrm{~cm}^{-3}\right)$ and $3.1 \%$ at
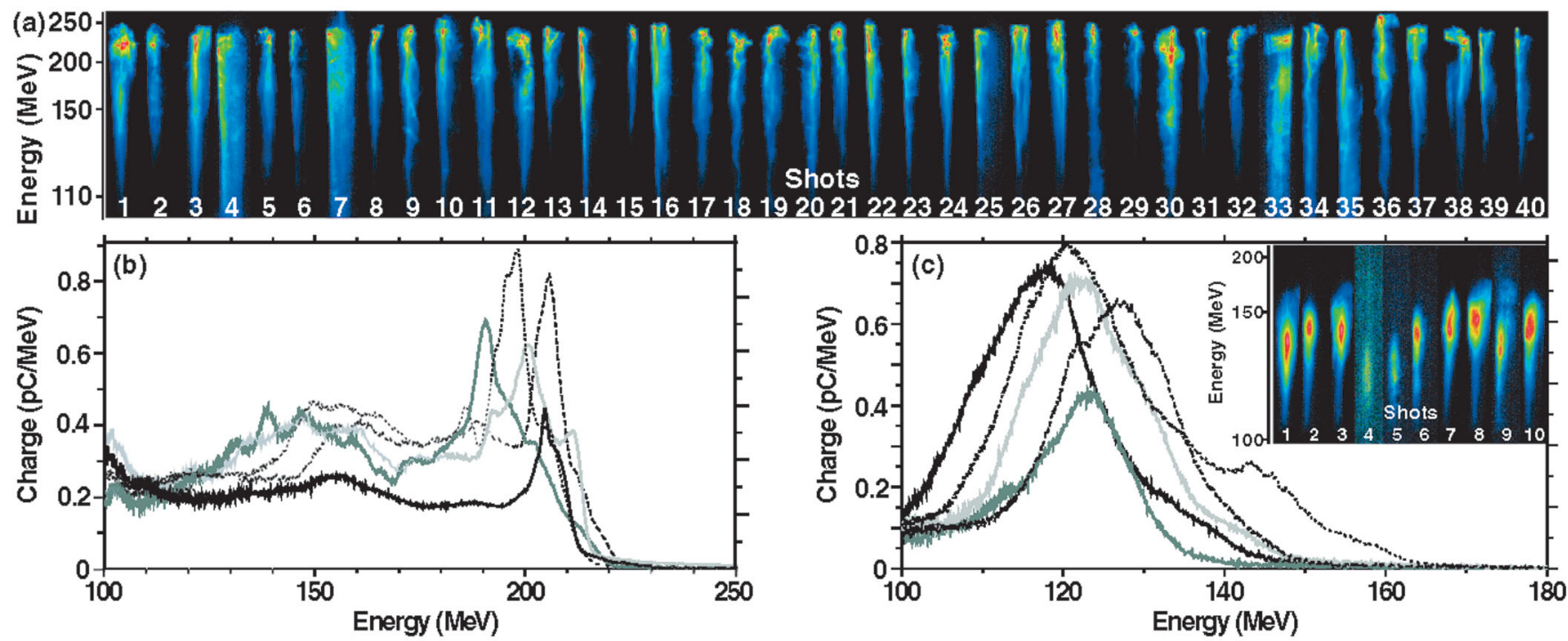

FIG. 3 (color). (a) False-color images of 40 consecutive, spatially dispersed electron beams on $S 2\left(n_{e} \approx 7.3 \times 10^{18} \mathrm{~cm}^{-3}\right)$ [sample spectra in (b)]. (c) Exemplary spectra for $n_{e} \approx 6.8 \times 10^{18} \mathrm{~cm}^{-3}$. Ten consecutive images of $S 2$ are presented in the inset. The color scale is normalized for each shot. 


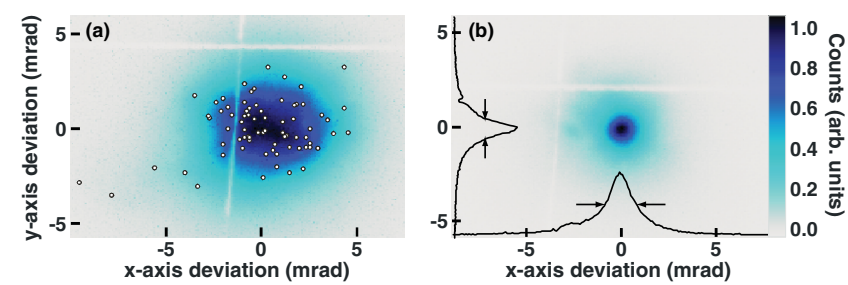

FIG. 4 (color). False-color images at $S 1$. (a) Summed signal of 74 electron beams and the peak positions of the individual shots (dots) at $n_{e} \approx 7.8 \times 10^{18} \mathrm{~cm}^{-3}$. rms shot-to-shot pointing fluctuations are 1.4 ( $y$ axis) and $2.2 \mathrm{mrad}$ ( $x$ axis). (b) Signal of a single electron beam with a FWHM divergence of 1.6 mrad.

$198 \mathrm{MeV}\left(n_{e}^{\prime} \approx 7.3 \times 10^{18} \mathrm{~cm}^{-3}\right)$. Therefore, the real energy stability might have been significantly more favorable than stated above. However, owing to low-angle scattering introduced by $S 1$, the pointing and energy spectra of an electron beam could not be measured simultaneously. Analyzing all shots from Fig. 4(a) individually yields a distribution of the electron bunch divergences. Figure 4(b) shows such a single electron beam imprint. The average beam divergence is $2.1 \pm 0.5 \mathrm{mrad}$ FWHM, or $0.9 \pm$ $0.2 \mathrm{mrad} \mathrm{rms}$ assuming a Gaussian profile. This divergence is smaller by almost a factor of 2 compared to other studies (e.g., [6]) and originates from collective electron dynamics, trapping, and intrabunch Coulomb repulsion, whereas the contribution of scattering off background particles is negligible [19].

We have performed 3D simulations of the laser-plasma interaction with the particle-in-cell code ILLUMINATION [20] to determine the role of pulse-shaping effects under our conditions. They indicate that electron trapping in the laser wake occurs only after the laser pulse has undergone significant self-focusing and -compression. This is supported by the fact that relativistic electrons were solely detected in the experiment for laser powers well above $P_{\text {crit }}$, which is exceeded for $n_{e}>2 \times 10^{18} \mathrm{~cm}^{-3}$ with ATLAS parameters. Therefore, the dramatic improvement in stability over our earlier work [7] (for a quantitative comparison cf. Ref. [21]) can be attributed to the modified gas target only. Previously, the transverse and longitudinal electron-density profile was preshaped by an electrical discharge and subsequent plasma cooling [22] allowing for guiding of the laser over several Rayleigh lengths. This facilitated longer acceleration distances and higher electron energies but may have introduced significant shotto-shot density-profile variations. The elimination of these fluctuations led to reproducible plasma conditions and electron beams in this study.

In conclusion, we have generated electron bunches from LWFA showing excellent stability by utilizing a hydrogen cell as the laser-plasma interaction volume. The virtue of our experiment lies in the improvement and simultaneous shot-to-shot stability of electron beam key parameters (divergence, pointing direction, charge, and energy). Such a stability will be of crucial importance to the success of laser-driven electron sources for applications such as the generation of undulator radiation [23] or free-electron lasers [9]. Further, this concept has the benefit of simplicity compared to other setups (e.g., [8]) and unlike gas jets or discharge waveguides can easily be scaled to very high repetition rates by employing continuous gas flow.

We acknowledge helpful discussions with K. Schmid, S. Rykovanov, and J. Meyer-ter-Vehn. This work was funded by the DFG through the TR18 and MAP schemes and by the Association EURATOM-MPI für Plasmaphysik. Zs. M. received financial help from the MarieCurie Individual Fellowship No. MEIF-CT-2005-024150 and B. H. was supported through DFG No. GRK1203.

*Corresponding author. stefan.karsch@mpq.mpg.de

[1] T. Tajima and J. M. Dawson, Phys. Rev. Lett. 43, 267 (1979).

[2] A. Modena et al., Nature (London) 377, 606 (1995).

[3] J. Faure et al., Nature (London) 431, 541 (2004); S. P. D. Mangles et al., Nature (London) 431, 535 (2004); C. G. R. Geddes et al., Nature (London) 431, 538 (2004).

[4] B. Hidding et al., Phys. Rev. Lett. 96, 105004 (2006).

[5] A. Pukhov and J. Meyer-ter-Vehn, Appl. Phys. B 74, 355 (2002).

[6] W.P. Leemans et al., Nature Phys. 2, 696 (2006); K. Nakamura et al., Phys. Plasmas 14, 056708 (2007).

[7] S. Karsch et al., New J. Phys. 9, 415 (2007).

[8] J. Faure et al., Nature (London) 444, 737 (2006).

[9] F. Grüner et al., Appl. Phys. B 86, R431 (2007).

[10] S. Gordienko and A. Pukhov, Phys. Plasmas 12, 043109 (2005).

[11] J. Faure et al., Phys. Rev. Lett. 95, 205003 (2005).

[12] D. C. Barnes, T. Kurki-Suonio, and T. Tajima, IEEE Trans. Plasma Sci. 15, 154 (1987).

[13] G.-Z. Sun et al., Phys. Fluids 30, 526 (1987).

[14] S. P. D. Mangles et al., Phys. Plasmas 14, 056702 (2007).

[15] T. Hosokai et al., Phys. Rev. E 67, 036407 (2003).

[16] J. Zierep and K. Bühler, Strömungsmechanik (Springer, Heidelberg, 1991).

[17] K. A. Tanaka et al., Rev. Sci. Instrum. 76, 013507 (2005).

[18] W.Lu et al., Phys. Rev. ST Accel. Beams 10, 061301 (2007).

[19] The probability of an electron scattering event to occur with a deflection angle $>100 \mu \mathrm{rad}$ (the CAWO screen resolution limit) is (a) $\sim 6 \times 10^{-3}$ inside the gas cell due to Mott scattering off hydrogen ions and Møller scattering off plasma electrons and (b) $<10^{-6}$ inside the vacuum chamber due to scattering off residual gas atoms.

[20] M. Geissler, J. Schreiber, and J. Meyer-ter-Vehn, New J. Phys. 8, 186 (2006).

[21] Compared to Ref. [7], the relative rms shot-to-shot bunch fluctuations improved in energy from $30 \%$ to $2.5 \%$, in charge from $75 \%$ to $16 \%$, in absolute pointing from 5.6 to $1.4 \mathrm{mrad}$, and in FWHM divergence from $3.0 \pm 1.2$ to $2.1 \pm 0.5 \mathrm{mrad}$.

[22] D. J. Spence and S. M. Hooker, Phys. Rev. E 63, 015401 (R) (2000).

[23] H.-P. Schlenvoigt et al., Nature Phys. 4, 130 (2008). 